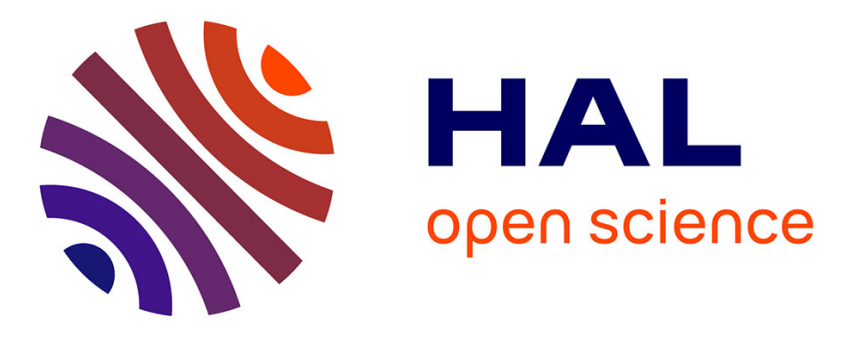

\title{
Continuous Transverse Stub Array for Ka-Band Applications
}

\author{
Mauro Ettorre, Francesco Foglia Manzillo, Massimiliano Casaletti, Ronan \\ Sauleau, Laurent Le Coq, Nicolas Capet
}

\section{- To cite this version:}

Mauro Ettorre, Francesco Foglia Manzillo, Massimiliano Casaletti, Ronan Sauleau, Laurent Le Coq, et al.. Continuous Transverse Stub Array for Ka-Band Applications. IEEE Transactions on Antennas and Propagation, 2015, 63 (11), pp.4792-4800. 10.1109/TAP.2015.2479243 . hal-01234327

\section{HAL Id: hal-01234327 \\ https://hal.sorbonne-universite.fr/hal-01234327}

Submitted on 30 Jul 2021

HAL is a multi-disciplinary open access archive for the deposit and dissemination of scientific research documents, whether they are published or not. The documents may come from teaching and research institutions in France or abroad, or from public or private research centers.
L'archive ouverte pluridisciplinaire HAL, est destinée au dépôt et à la diffusion de documents scientifiques de niveau recherche, publiés ou non, émanant des établissements d'enseignement et de recherche français ou étrangers, des laboratoires publics ou privés.

\section{(c)(1)}

Distributed under a Creative Commons Attribution| 4.0 International License 


\title{
Continuous Transverse Stub Array for Ka-Band Applications
}

\author{
Mauro Ettorre, Senior Member, IEEE, Francesco Foglia Manzillo, Student Member, IEEE, \\ Massimiliano Casaletti, Member, IEEE, Ronan Sauleau, Senior Member, IEEE, Laurent Le Coq, and Nicolas Capet
}

\begin{abstract}
This paper presents a flat, high gain, wide scanning, broadband continuous transverse stub (CTS) array. The design procedure, the fabrication, and an exhaustive antenna characterization are described in details. The array comprises 16 radiating slots and is fed by a corporate-feed network in hollow parallel plate waveguide (PPW) technology. A pillbox-based linear source illuminates the corporate network and allows for beam steering. The antenna is designed by using an ad hoc mode matching code recently developed for CTS arrays, providing design guidelines. The assembly technique ensures the electrical contact among the various stages of the network without using any electromagnetic choke and any bonding process. The main beam of the antenna is mechanically steered over $\pm 40^{\circ}$ in elevation, by moving a compact horn within the focal plane of the pillbox feeding system. Excellent performances are achieved. The features of the beam are stable within the design $27.5-31 \mathrm{GHz}$ band and beyond, in the entire Ka-band (26.5-40 GHz). An antenna gain of about $29 \mathrm{dBi}$ is measured at broadside at $29.25 \mathrm{GHz}$ and scan losses lower than $2 \mathrm{~dB}$ are reported at $\pm 40^{\circ}$. The antenna efficiency exceeds $80 \%$ in the whole scan range. The very good agreement between measurements and simulations validates the design procedure. The proposed design is suitable for Satcom Ka-band terminals in moving platforms, e.g., trains and planes, and also for mobile ground stations, as a multibeam sectorial antenna.
\end{abstract}

Index Terms-Broadband antennas, multibeam antennas, parallel plate waveguide (PPW), slot arrays, waveguide arrays.

\section{INTRODUCTION}

$\mathbf{T}$ HE WORLDWIDE increasing demand for broadband satellite communication systems has led to the deployment of satellite constellations in Ka-band and development of novel user terminals for fixed and moving users [1]. The allocated bands for both military and civil applications for receiving (Rx) and transmitting (Tx) units are 17.7-21.2 GHz and 27.5$31 \mathrm{GHz}$, respectively. In both bands, stringent requirements are imposed on the antenna part. The antenna should present a

M. Ettorre, F. Foglia Manzillo, R. Sauleau, and L. Le Coq are with the Institute of Electronics and Telecommunications of Rennes (IETR), UMR CNRS 6164, University of Rennes 1, 35042 Rennes, France (e-mail: mauro.ettorre@univ-rennes1.fr).

M. Casaletti is with Sorbonne Universités, UPMC Université Paris 06, F-75005 Paris, France (e-mail: massimiliano.casaletti@upmc.fr).

N. Capet is with the Antenna Department, Centre National d'Etude Spatiale (CNES), 31401 Toulouse, France (e-mail: nicolas.capet@cnes.fr). high gain, and operate in circular polarization over an angular sector covering the full azimuthal plane and a wide angle in elevation. The masks for the radiated beams are prescribed by the standards of European Telecommunications Standard Institute (ETSI), Federal Communications Commission (FCC), and International Telecommunication Union (ITU), depending on the operating country and satellite operator. In addition, the antenna should be flat, compact, and low weight for aesthetic and aerodynamic needs, but at the same time mechanically robust.

Lately, several solutions have been proposed trying to fulfill all previous requirements. In particular, we can mention classical parabolic systems [2], electromechanical scanning planar antenna arrays [3], [4], phased arrays [1], [5], and lately promising metamaterial-based solutions [6]. In all cases, the driving features of the antenna development have been cost, efficiency, compactness, and impact of the antenna structure on the fuselage of the moving platform. For these reasons, electromechanical or fully mechanical scanning schemes [3], [4] have been preferred to more advanced but costly and lossy phased array solutions [1], [5] or bulky but low-cost parabolic systems [2]. However, there is still a clear need for novel concepts to further reduce the profile and footprint of the antenna at a lower cost and improved radiation performance.

Arrays of continuous transverse stubs (CTSs) can be considered a good candidate for advanced antenna systems due to their attractive performance and fabrication stability [7]. This latter aspect is particularly important for cost reduction especially in Ka-band where high fabrication accuracies drive the overall cost of the antenna. CTS arrays consist of broad stubs, finite in height, connected to a parallel plate waveguide (PPW) feeding system, and radiating in free space [8]-[12]. The radiating stubs can be fed in parallel or in series by the PPW structure. In particular, when the stubs are fed in parallel, the CTS array presents a very wide bandwidth and a wide scanning capability, unparalleled by other antenna solutions [11]. CTS arrays operate in linear polarization based on their radiation mechanism. A circular polarization can be achieved by using classical polarizers [11]. It is worth mentioning that CTS arrays may look like connected arrays of slots [13], [14]. However, connected arrays of slots need several feeds in a double mesh grid with a periodicity lower than half-wavelength at the upper frequency of operation of the antenna. In addition, a backing ground reflector is needed for unidirectional radiation and increased efficiency.

Attracted by the very appealing features of CTS antennas, we have recently developed a fast mode matching code for their analysis and design [15]. In this paper, this code has been used 


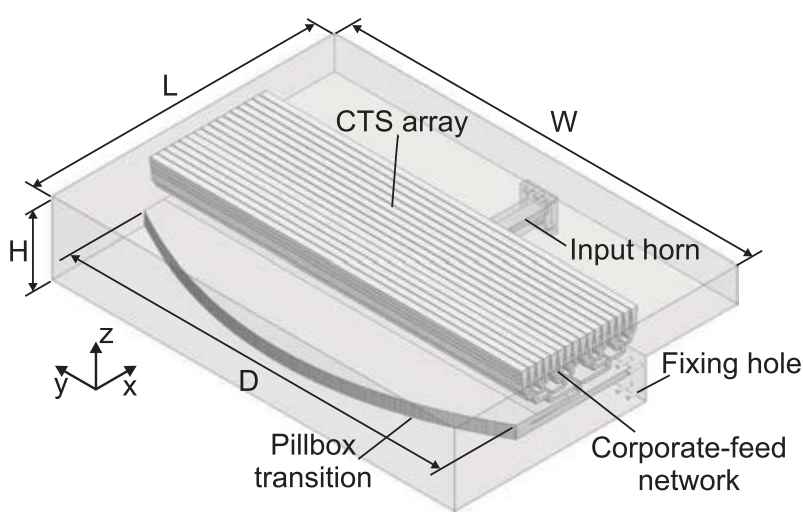

(a)

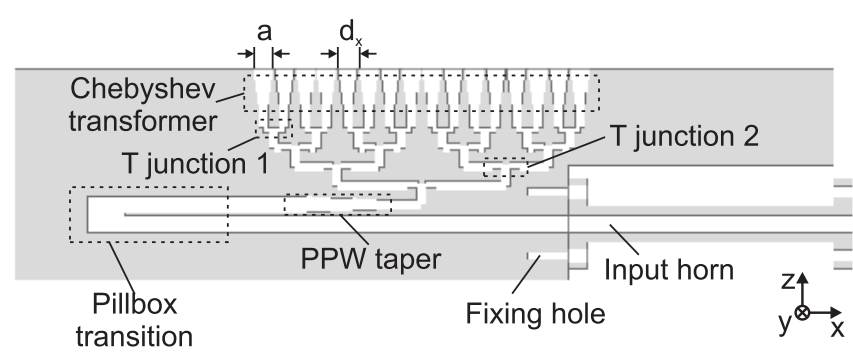

(b)

Fig. 1. CTS array. (a) Perspective view. (b) Cross-sectional view taken along the $x z$-plane. The various building blocks are highlighted. $a$ and $d_{x}$ denote the slot width and slot periodicity, respectively.

to design an array of 16 slots (stubs of zero height) operating in the 27.5-31 GHz band (Tx Satcom band) and fed in parallel by a corporate-feed network in PPW technology, as shown in Fig. 1. This feed network matches the active impedance of the CTS array to the input PPW. The linear source needed for the CTS array is a critical part. It has been designed for steering mechanically the antenna main beam and is based on a pillbox transition as in [16]. A target scan range of $\pm 30^{\circ}$ in H-plane has been chosen for the design. The pillbox transition is illuminated by a classical H-plane sectoral horn moving in its focal plane.

The final structure is in aluminum and has been fabricated in several pieces using standard milling and wire electric discharge machining (EDM) processes. The employed assembly technique overcomes the drawbacks of standard fabrication methods for CTS arrays [17], [18]. Indeed, the various sections of the antenna are screwed together and the electrical contact is preserved without any bonding layer or electromagnetic choke. Similar approaches have been used to manufacture less complex antenna structures at millimeter waves [19], [20]. The final thickness of the antenna is $45 \mathrm{~mm}$ and could be further reduced by using a dielectric-filled PPW corporate-feed network. The reported measured antenna performance is very promising and reveals the possibilities offered by CTS array for Satcom applications.

This paper is organized as follows. Section II summarizes the results in [15] in a design perspective, providing the requested slot size and periodicity. In Section III, the corporate-feed network is presented and the design of the various parts of the network is detailed. The fabrication procedure, prototype, and measurements are illustrated in Section IV. Finally, conclusion is drawn in Section V.

\section{CTS ARRAY: ANALYSIS AND DESIGN}

The radiation performance of a CTS array is dictated by the slot width $a$ and the array periodicity $d_{x}$ (refer to Fig. 1). The choice of the slot width is subject to several tradeoffs. The upper bound for $a$ is chosen in such a way to ensure a monomodal transverse electromagnetic (TEM) propagation regime over the entire frequency range of operation, yielding $a<$ $a_{\text {max }}=c / 2 f_{c}$, with $c$ the speed of light in free space and $f_{c}$ the cut-off frequency of the first higher order mode supported by a PPW [21]. By setting $f_{c}=31 \mathrm{GHz}$, i.e., the highest frequency in the targeted band, the constraint $a<a_{\max }=4.84 \mathrm{~mm}$ is obtained. On the other hand, as discussed in [15] and shown later in brief, for a given slot periodicity $d_{x}$, the real part of the active slot impedance $Z_{a c t}$ in the H-plane of a CTS array increases when $a$ decreases. Therefore, small values of $a$ will cause severe impedance mismatches between the slot impedance and the feeding PPW, typically of height $a$. In addition, small slot widths result in larger losses in the feeding PPWs [21].

Another aspect to take into account in choosing the periodicity $d_{x}$ and the size of the slots $a$ is the location of the scan plane. In the present case, the CTS array is designed to steer its beam only in the H-plane ( $y z$-plane in Fig. 1), i.e., where the long side of the slots lies. In this plane, no grating lobes can occur for array periodicity lower than the free-space wavelength. Thus, there are not strict constraints on the inter-element spacing $d_{x}$. However, large array spacings result in poor matching characteristics, since $a$ cannot be increased beyond $a_{\max }$. On the other hand, tightly spaced slots make harder the design of the corporate-feed network along the $x$-direction. Based on this discussion, the array periodicity has been fixed to $d_{x}=4.5 \mathrm{~mm}$ as a good compromise between spacing among slots and matching capabilities.

In Fig. 2, the active slot impedance of an infinite CTS array with this value for $d_{x}$ has been computed for different slot widths $a$, approaching its upper bound $a_{\max }$, by using the numerical model presented in [15]. Each active impedance is normalized with respect to the characteristic impedance $Z_{0}=$ $\eta a / w$ of a PPW line having its height equal to the slot width $a$ and an unitary length $w$ ( $\eta$ is the free-space impedance). Two different pointing directions in the H-plane are considered: $\theta=0^{\circ}$ in Fig. 2(a) and $\theta=45^{\circ}$ in Fig. 2(b). Note that the latter value exceeds the targeted field of view of $\left(-30^{\circ}, 30^{\circ}\right)$. The results demonstrate that for $d_{x}=4.5 \mathrm{~mm}$, the active impedance experiences limited variations when the array scans in the H-plane, for all considered slot widths.

Finally, the value $a=0.83 a_{\max }=4 \mathrm{~mm}$ is chosen for the slot width, so that the cut-off frequency of the first higher order mode is set to $37.5 \mathrm{GHz}$, i.e., sufficiently far from the maximum operating frequency to practically ensure a TEM propagation. Besides, a good mechanical robustness of the CTS array can be achieved in the last section of the corporate-feed network. The active impedance for the design values of $a$ and $d_{x}$ is plotted in Fig. 3 in Ka-band for different beam pointing directions 


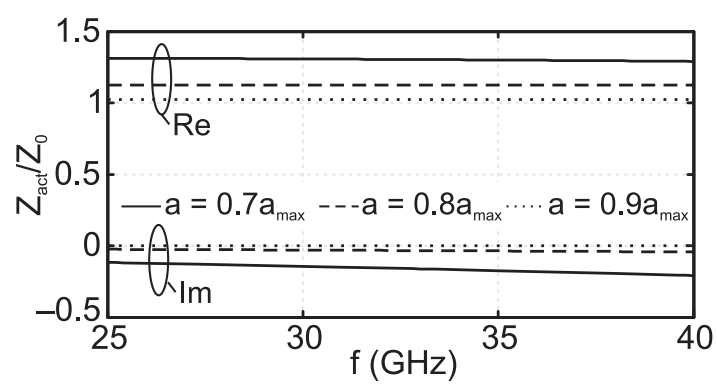

(a)

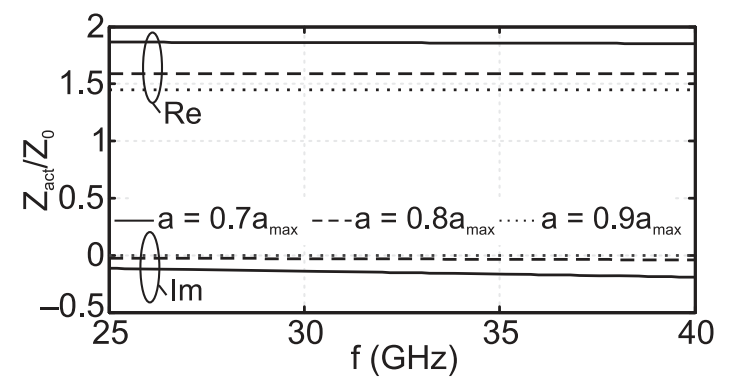

(b)

Fig. 2. Normalized active slot impedance of an infinite CTS array with spacing $d_{x}=4.5 \mathrm{~mm}$ for several slot widths, when the beam is steered in the H-plane. (a) $\theta=0^{\circ}$. (b) $\theta=45^{\circ}$. The impedance is normalized to $Z_{0}=\eta a / w$ whereas the slot widths to $a_{\max }=4.84 \mathrm{~mm}$. Re and Im indicate the real and imaginary part of the slot active impedance, respectively.

in the H-plane. The real part of $Z_{a c t}$ is almost constant versus frequency, thus providing the broadband behavior of the array. The imaginary part is much smaller than the characteristic impedance $Z_{0}$ of the feeding line and can be compensated by the corporate-feed network. The active impedance keeps quite stable even when the array beam is steered in the H-plane, thus allowing the antenna module to achieve excellent scanning performance.

The real part increases by a factor 1.2 for scan angles in the range $\left(-30^{\circ}, 30^{\circ}\right)$ and by a factor 1.5 in the range $\left(-45^{\circ}, 45^{\circ}\right)$. Note that in the latter range, the maximum value of $\operatorname{Re}\left(Z_{a c t}\right)=$ $1.6 Z_{0}$. The reference PPW line employed in the design of the corporate-feed network has a characteristic impedance equal to $Z_{0} / 2$, as discussed in Section III. Therefore, the maximum impedance ratio occurring in the first section of the feed network is 1:3.2. For this reason, a two-section Chebyshev transformer is proposed in the next section to obtain a wide band matching between the slot active impedance and the corporate-feed network.

\section{CORPorAte-FEED NETWORK}

In this section, we present the design of each part of the corporate-feed network. Circuit models have been used in conjunction with the numerical method proposed in [15] for defining each building block [22]. For brevity, only the final geometrical parameters of the various parts are provided. However, full-wave numerical results are compared in Section IV with measurements for the final antenna structure, validating the design. The corporate-feed network operates in the fundamental TEM mode of the associated hollow PPW

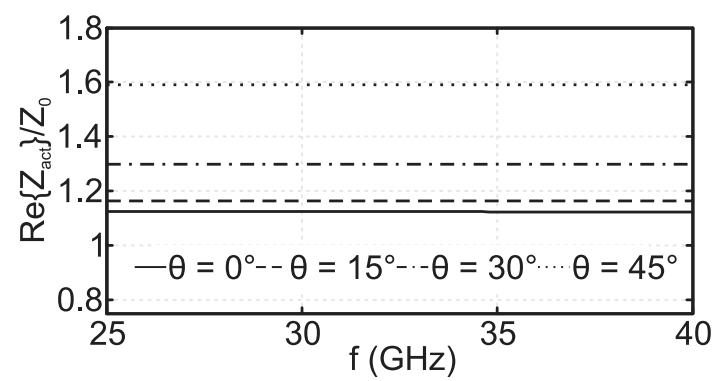

(a)

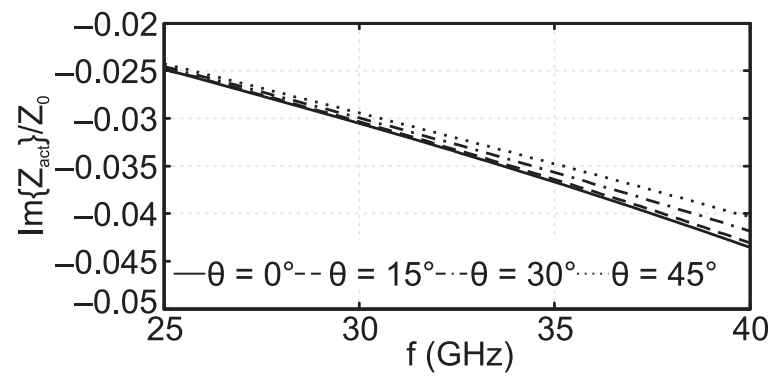

(b)

Fig. 3. Normalized active slot impedance of an infinite CTS array with $d_{x}=$ $4.5 \mathrm{~mm}$ and $a=4.0 \mathrm{~mm}$ for different pointing directions in the H-plane, $\theta=$ $\left(0^{\circ}, 15^{\circ}, 30^{\circ}, 45^{\circ}\right)$. (a) Real part of $Z_{a c t}$. (b) Imaginary part of $Z_{a c t}$. The active impedance is normalized to $Z_{0}=\eta a / w$.

lines. The design frequency is fixed to $f_{0}=29.25 \mathrm{GHz}$ with a free-space wavelength of $\lambda_{0}=10.26 \mathrm{~mm}$. Each part of the feed network is optimized to achieve an input reflection coefficient lower than $-30 \mathrm{~dB}$ at its input port at the design frequency for broadside radiation.

\section{A. Chebyshev Transformer}

The two-section Chebyshev transformer shown in Fig. 4(a) has been used to match the active impedance of the slots of the CTS array to the feeding PPW line. The PPW line has a height equal to $2 \mathrm{~mm}$. Such a value has been chosen as a tradeoff between the total thickness of the structure ( $H$ in Fig. 1$)$ and the possibility to allocate the feeding line between two adjacent slots, as shown in Fig. 4(a). The procedure outlined in [23] has been used for designing the transformer. The design of the transformer assumes a single-mode propagation. Higher order modes generated at the slot level are considered attenuated after the first PPW section of length $2.35 \mathrm{~mm}$ and height $a$ connecting the radiating slot to the transformer. The maximum reflection coefficient and the ratio between the load and characteristic impedances at the output and input ports have been chosen equal to 0.05 and 2 , respectively. The final design parameters are shown in Fig. 4(a).

\section{B. E-Plane T-Junction I}

This E-plane T-junction [Fig. 4(b)] is used to match the input impedance of two consecutive slots seen at the output ports of the Chebyshev transformer to the input PPW feeding line of height $2.00 \mathrm{~mm}$, as shown in Fig. 4(b). The input and output ports present the same profile and thus the same characteristic impedance. Therefore, a combination of height change at the 


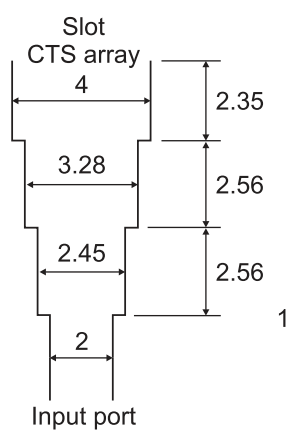

(a)

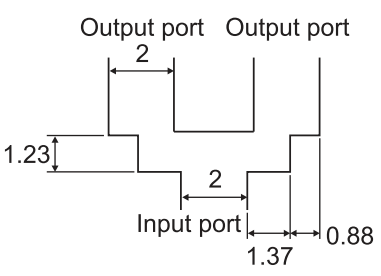

(b)

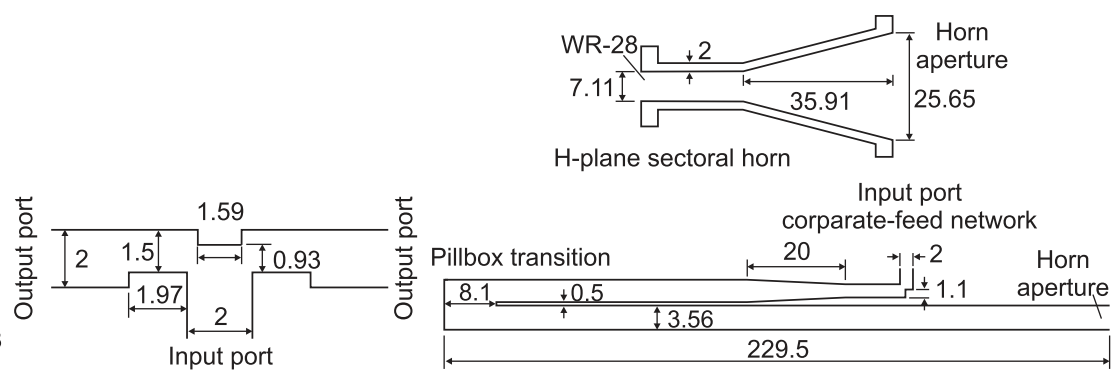

(c)

(d)

Fig. 4. Cross sectional view along the $x z$-plane of the building blocks of the corporate-feed network and top view of the H-plane sectoral horn. (a) Chebyshev transformer. (b) E-plane T-junction 1. (c) E-plane T-junction 2. (d) Pillbox transition and H-plane sectoral horn. All dimensions are given in millimeters.

center part of the junction and reactive loading at the $90^{\circ}$ bends has been used for matching. The final design parameters are reported in Fig. 4(b).

\section{E-Plane T-Junction 2}

In this case [Fig. 4(c)], a classical power divider in PPW technology is associated to a quarter wavelength transformer for matching the output ports to the input one over a wide bandwidth [11]. The input and output ports have the same characteristic impedance and height $(2.00 \mathrm{~mm})$. The quarterwavelength transformer of height $1.50 \mathrm{~mm}$ is used to halve the impedance value at the output ports before joining the section with height $0.93 \mathrm{~mm}$. A matching is therefore achieved, since the output ports are seen in series by the input port. The final geometrical size is listed in Fig. 4(c).

\section{Pillbox Transition}

The pillbox transition [Fig. 4(d)] creates the line source needed to excite the CTS array. It is made by two stacked PPW lines coupled by a long slot of width $8.1 \mathrm{~mm}$ contouring a two-dimensional (2-D) parabolic reflector in the common metal plate between the two PPW lines of thickness $0.5 \mathrm{~mm}$, as shown in Fig. 4(d). The pillbox system converts the cylindrical TEM mode launched by the H-plane sectoral horn in the lower PPW line into a plane one, traveling in the upper PPW line and afterward in the corporate-feed network [24]. The guidelines provided in [16] and [24] have been followed for designing the pillbox transition. The parabolic reflector has a focal distance equal to $F=10 \lambda_{0}=102.6 \mathrm{~mm}$ and a diameter of $D=22.38 \lambda_{0}=229.5 \mathrm{~mm}$. The transition is illuminated by a H-plane sectoral horn providing an edge tapering of $-30 \mathrm{~dB}$. The horn aperture is equal to $2.5 \lambda_{0}=25.65 \mathrm{~mm}$ and is fed by a WR-28 waveguide with inner size equal to $7.11 \times 3.56 \mathrm{~mm}^{2}$ thanks to a transition of length $35.91 \mathrm{~mm}$. The horn is made in aluminum with a wall thickness of $2.00 \mathrm{~mm}$. A smooth transition of length $20 \mathrm{~mm}$ matches the upper PPW line of the pillbox system to the PPW line used for the corporate-feed network with a height of $2.00 \mathrm{~mm}$. Finally, a $90^{\circ}$ bend with a reactive step of height $1.10 \mathrm{~mm}$ connects the pillbox transition to the input port of the corporate-feed network [refer to Fig. 4(d)]. The antenna main beam is steered in H-plane by moving the input horn in the focal plane of the pillbox transition. For this reason, the lower PPW line of the pillbox transition is left open on the edge connected to the horn and several holes are made on this side of the antenna for fixing the horn, as shown in Fig. 1.

\section{Prototype And Measurements}

The antenna design described in the previous sections has been realized in aluminum. An effective procedure based on the fabrication of separate modules and their assembly by means of screws has been employed to ease the manufacturing. Indeed, standard methods to fabricate hollow CTS arrays rely on costly bonding or brazing processes [18]. Corrugations and adhesive layers are used to ensure a good electric contact through the CTS array, but they may introduce air-gaps, thus lowering the antenna performance. For the present design, the corporate-feed network and pillbox system have been divided in nine parts (P1-P9), as shown in Fig. 5. Each part has been manufactured as a stand-alone element using either a milling or EDM process. The central part of the corporate-feed network is made by P3-P6. These parts are assembled by sliding them in P2. The ensemble is fixed to P1 using dowels and then connected to P7. At the same time, P8 and P9 are joined together with dowels and slid into P7. Finally, screws are used on the lateral and bottom sides of the antenna to fix all parts. An alignment accuracy of about $10 \mu \mathrm{m}$ is guaranteed by the dowels. A fabrication accuracy better than $50 \mu \mathrm{m}$ is expected. The horn has been realized in a single piece by using an EDM process. The wedges on the horn aperture, as shown in Fig. 5, are used for a better alignment with the lower PPW line of the antenna structure. Complementary wedges are made in P7 and P9. The final antenna prototype and feeding horn are shown in Fig. 6. The horn is fixed to the antenna by screws using the holes shown in Fig. 6 located in P7 and P9. Analogous fabrication techniques have been adopted for other antenna modules operating at millimeter waves [19], [20]. However, to the best of authors' knowledge, no CTS arrays presented in open literature have been implemented with this approach. The assembling solution does not employ any bonding process and does not require any electromagnetic choke among the various stages of the hollow corporate-feed network as in previous CTS designs [17], [18], thus reducing the complexity and cost of the overall structure. 


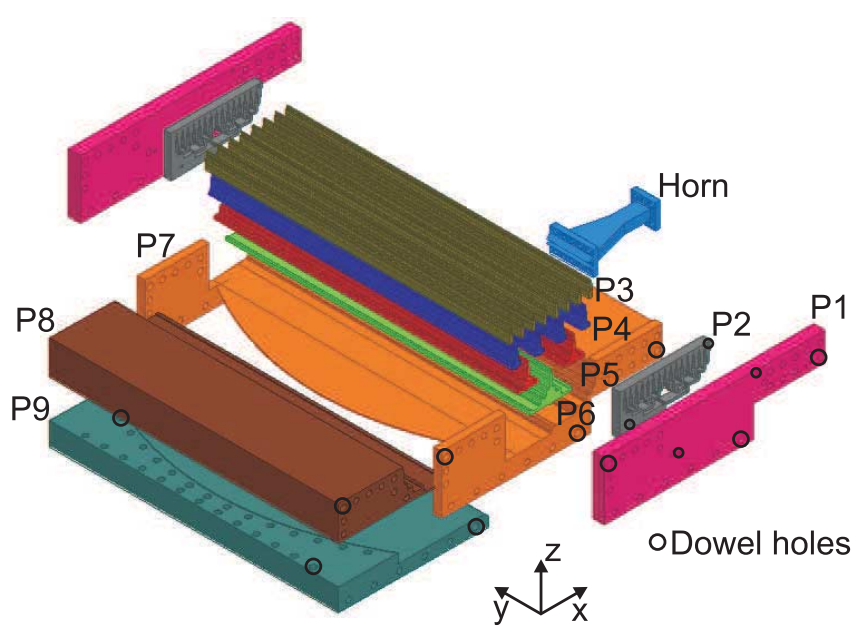

Fig. 5. Manufacturing and assembling details. The various parts of the antenna are aligned with dowels and screwed together. Black circles indicate the positions of the dowels on one lateral side of the antenna. For symmetry, dowel holes are present also on the other side.

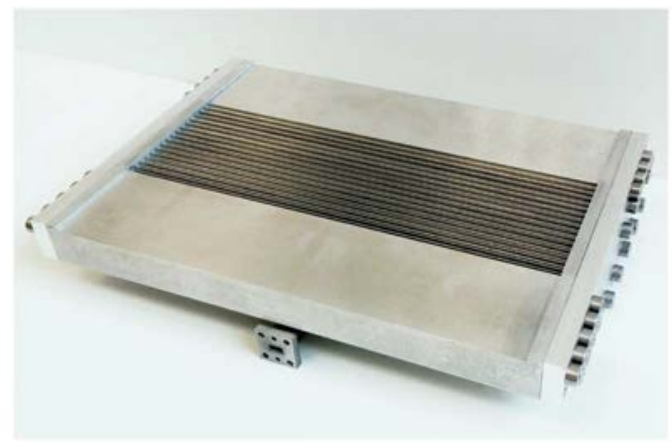

(a)

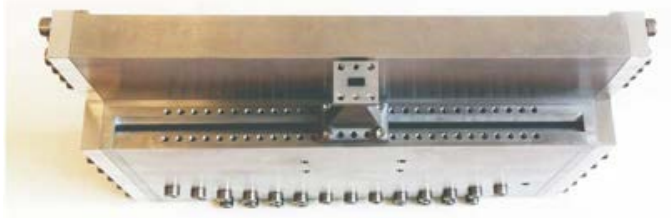

(b)

Fig. 6. Antenna prototype. (a) Three-dimensional (3-D) view. The CTS array and feeding horn can be recognized. (b) Lateral view from the feeding side.

The prototype has been measured at IETR. Different horn positions in the focal plane of the pillbox transition have been considered to evaluate the antenna performance in scanning. The step between two consecutive horn positions is equal to $1.25 \lambda_{0}$, corresponding to half the size of the horn aperture. The central position is indicated with Pos. 0 and ascending numbers are used for lateral positions along the positive $y$-axis. The antenna performance is reported only for positive displacements of the feeding horn along the $y$-direction. Indeed, since the electromagnetic design is symmetric, similar results are obtained for a feed placed in the negative $y$-direction. We recall that the antenna has been designed for a maximum scan angle in elevation of about $30^{\circ}$ which corresponds to Pos. 4 in the following.

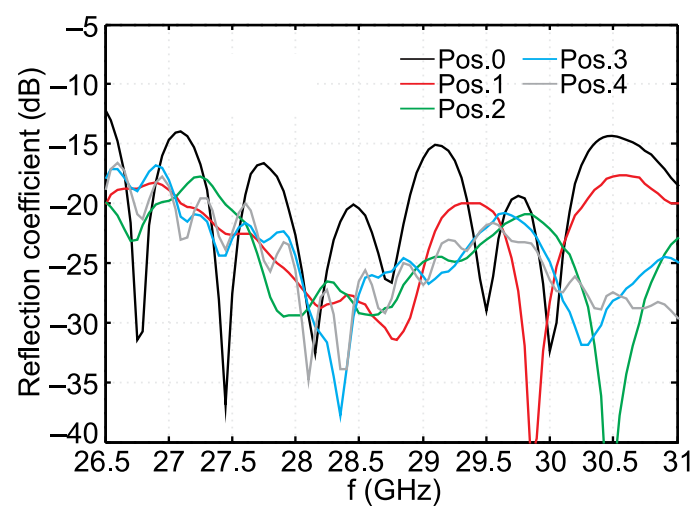

Fig. 7. Measured input reflection coefficients of the CTS array for several positions of the input horn in the focal plane of the pillbox transition.

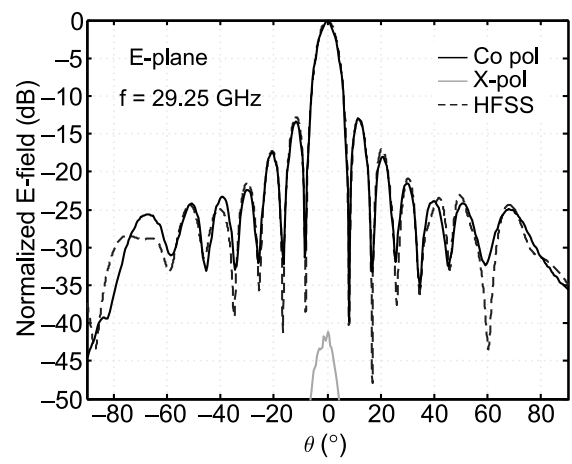

(a)

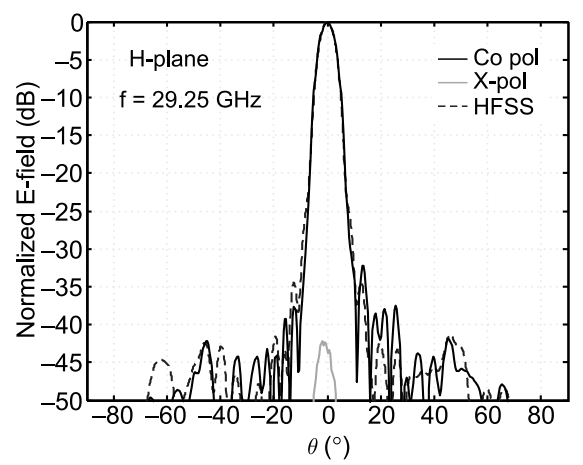

(b)

Fig. 8. Normalized radiation patterns at the design frequency for broadside radiation. (a) E-plane. (b) H-plane.

The measured input reflection coefficients of the CTS array for various positions in the band $26.5-31 \mathrm{GHz}$ are shown in Fig. 7. The antenna is matched in the overall considered band. Besides, an input reflection coefficient better than $-15 \mathrm{~dB}$ is obtained in the $27-31 \mathrm{GHz}$ band for all scanning positions. For brevity, only experimental results are provided, but the measurements are in very good agreement with full-wave simulation results.

The radiation pattern in the E-plane ( $x z$-plane in Fig. 1) and H-plane (yz-plane in Fig. 1) at the design frequency for broadside radiation (Pos.0) is reported in Fig. 8. An excellent agreement between measurements and simulations is obtained in both the E- and $\mathrm{H}$-plane. The measured $-3 \mathrm{~dB}$ beamwidths are $7.08^{\circ}$ and $6^{\circ}$ in E- and H-plane, respectively. In E-plane, 


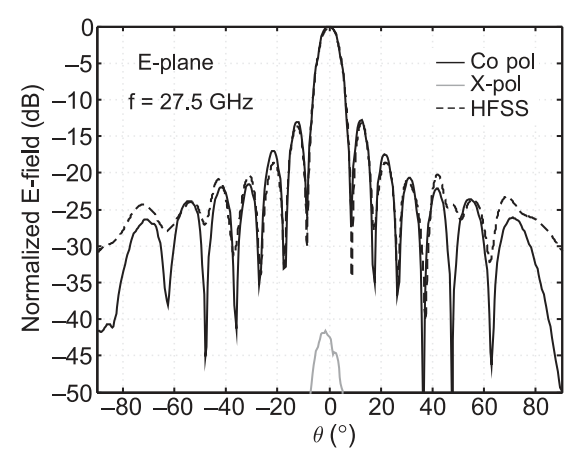

(a)

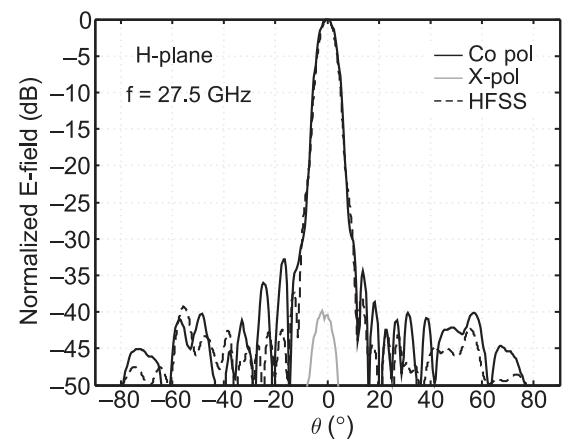

(b)

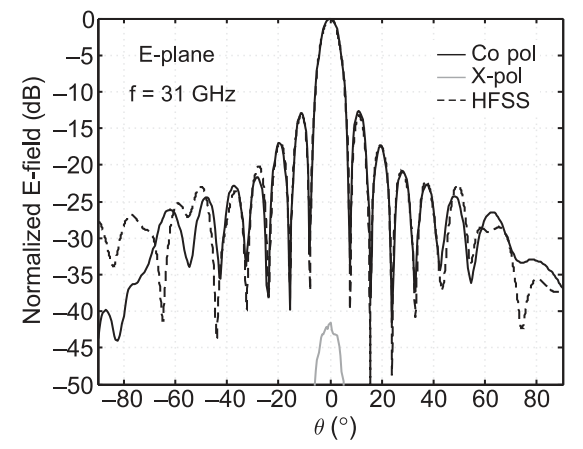

(c)

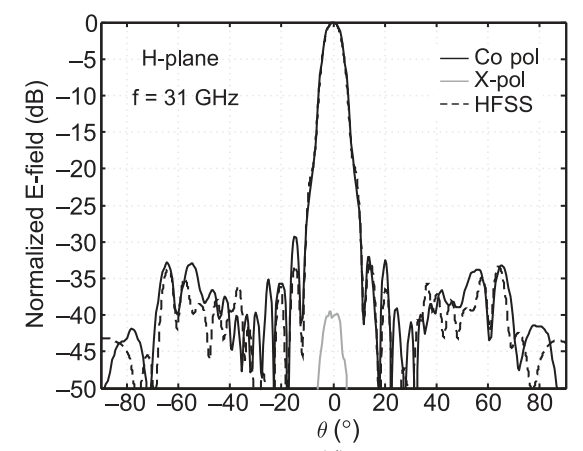

(d)

Fig. 9. Normalized radiation patterns at 27.5 and $31 \mathrm{GHz}$ for broadside radiation. (a) and (b) E- and H-plane patterns at $27.5 \mathrm{GHz}$. (c) and (d) E- and H-plane patterns at $31 \mathrm{GHz}$.

the sidelobe level (SLL) is limited by the uniform illumination of the corporate-feed network to values around $-13.26 \mathrm{~dB}$. Lower SLLs can be achieved by designing the corporate-feed network in such a way to provide a nonuniform illumination over the radiating aperture in E-plane. On the other hand, the SLL in H-plane is lower than $-30 \mathrm{~dB}$ thanks to the tapered

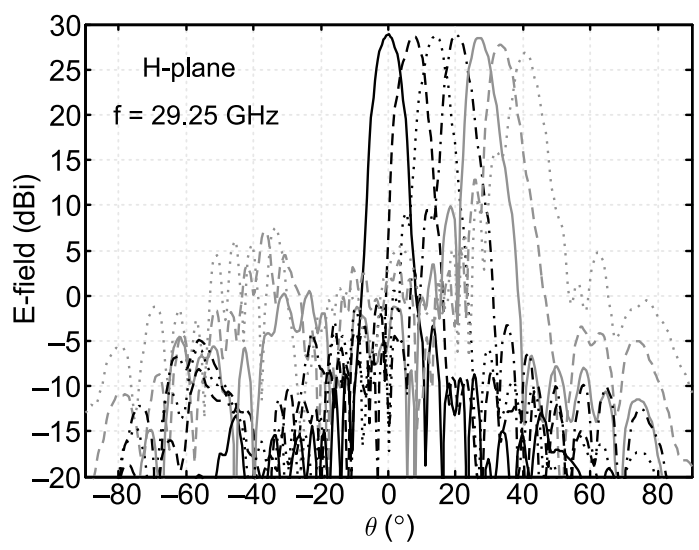

Fig. 10. Measured radiation patterns at the design frequency for various scan angles along the H-plane.

TABLE I

H-Plane Measured Radiation Performance of the CTS Array at THE DESIGN FREQUENCY

\begin{tabular}{|c|c|c|c|}
\hline Pos. & $\theta_{\max }\left(^{\circ}\right)$ & Gain $(\mathrm{dBi})$ & SLL $(\mathrm{dB})$ \\
\hline 0 & 0 & 28.87 & -32.20 \\
\hline 1 & 7.5 & 28.61 & -29.72 \\
\hline 2 & 14 & 28.77 & -19.56 \\
\hline 3 & 20 & 28.84 & -19.33 \\
\hline 4 & 27 & 28.52 & -18.66 \\
\hline 5 & 33 & 27.8 & -15.03 \\
\hline 6 & 40.5 & 26.85 & -10.95 \\
\hline
\end{tabular}

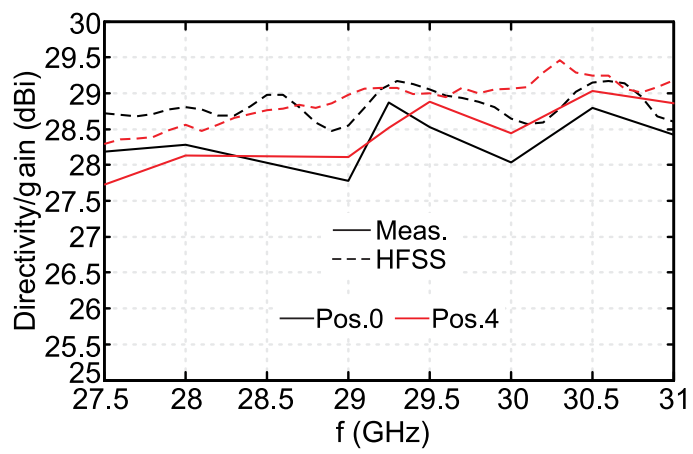

Fig. 11. Measured gain and simulated directivity in the band $27.5-31 \mathrm{GHz}$ for positions Pos.0 and Pos.4.

illumination provided by the pillbox transition. The measured cross-polarization levels are lower than $-40 \mathrm{~dB}$ (peak-to-peak) in both planes.

The radiation patterns at 27.5 and $31 \mathrm{GHz}$ are shown in Fig. 9 in E- and H-plane. An excellent agreement is obtained between simulations and measurements. Besides, the radiation performance at the design frequency is preserved within the frequency band of the CTS array showing the stable radiation properties of the antenna.

The measured radiation patterns along the $\mathrm{H}$-plane for different horn positions are shown in Fig. 10. The radiation performance for each considered horn position is summarized in Table I. A scan range beyond the design maximum angle of $30^{\circ}$ has been considered to further highlight the excellent scanning performance of the antenna. The SLLs are lower than $-18.6 \mathrm{~dB}$ within the $\pm 27^{\circ}$ scan range at $29.25 \mathrm{GHz}$ with scan 


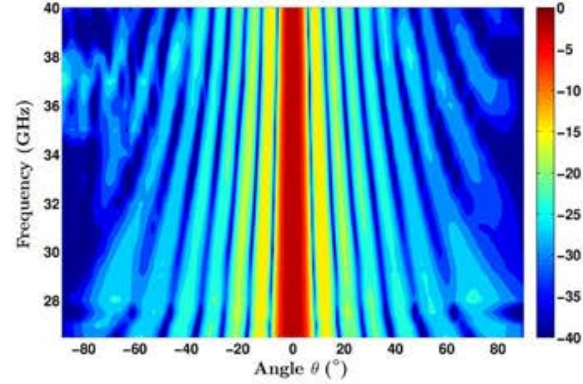

(a)

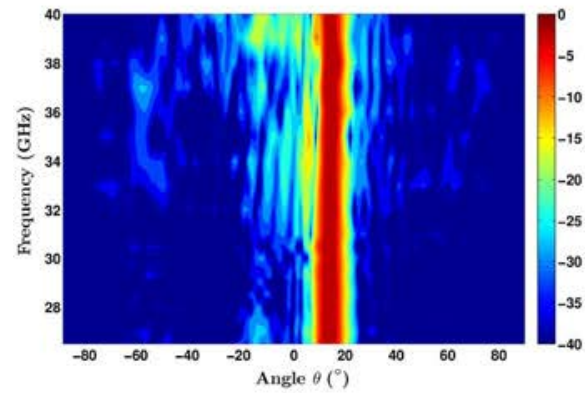

(d)

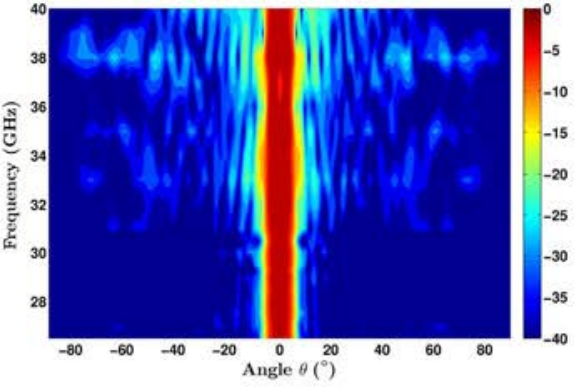

(b)

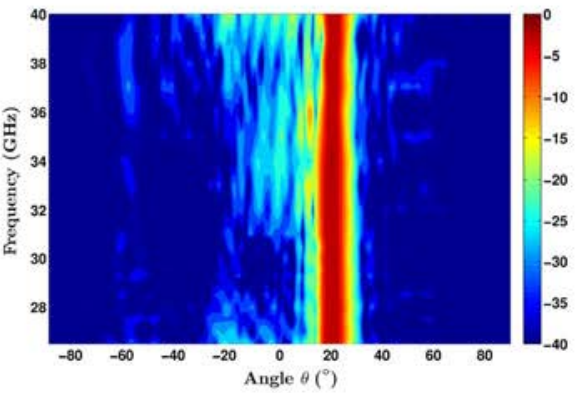

(e)

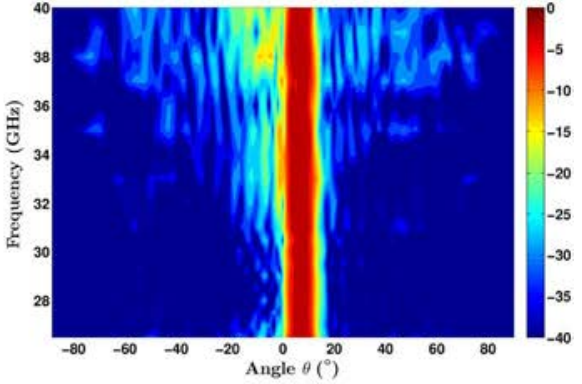

(c)

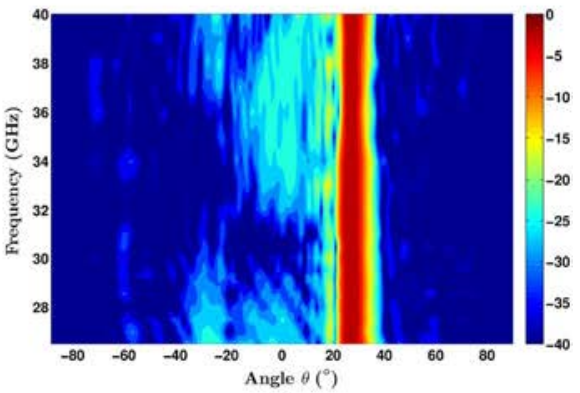

(f)

Fig. 12. Measured radiation patterns for different horn positions in the Ka-band, as functions of frequency and elevation angle. Patterns are normalized and plotted in dB. (a) E-plane, Pos.0. (b) H-plane, Pos.0. (c)-(f) H-plane, Pos.1-Pos.4.

losses lower than $0.35 \mathrm{~dB}$, whereas the SLL and the scan losses reduce to $\approx-11$ and $-2.02 \mathrm{~dB}$, respectively, for $\theta=40^{\circ}$.

The measured gain is compared to the simulated directivity in Fig. 11 for Pos.0 and Pos.4 (corresponding to extreme design scan angles) in the band $27.5-31 \mathrm{GHz}$. At the design frequency, the gain is $28.87 \mathrm{dBi}$ at broadside and $28.52 \mathrm{dBi}$ at $\theta=27^{\circ}$. Moreover, the gain is larger than $27.6 \mathrm{dBi}$ in the whole frequency band of operation for both feed positions. The antenna efficiency is equal to $93 \%$ at broadside at the design frequency. In the entire band, the efficiency is larger than $80 \%$ within the complete scan range. Even if not reported, similar results are obtained for other pointing directions between these two cases. These very good scanning performances are mainly due to the accurate design of the pillbox system and to the high edge tapering chosen for its parabolic reflector [16], as discussed in Section III-D.

Finally, the radiation patterns of the antenna in the entire Ka-band (26.5-40 GHz) have been measured and are shown in Fig. 12 for several horn positions covering the scan range $0^{\circ}-30^{\circ}$ (Pos.0-Pos.4). The E-plane radiation pattern is also provided for broadside radiation, clearly showing the wideband operation of the corporate-feed network. In the H-plane, the pointing direction is preserved within the overall band for all considered positions and the $-3 \mathrm{~dB}$ beamwidths have small variations.

\section{CONCLUSION}

A wideband, wide scanning, CTS array of 16 slots and fed in parallel by a corporate-feed network has been proposed as a very attractive solution for Ka-band applications like Tx or $\mathrm{Rx}$ units for Satcom terminals. The antenna operates in linear polarization but classical polarizers can be used to extend its operation to circular one. The main beam of the antenna is steered mechanically by moving an H-plane sectoral horn in the focal plane of the pillbox transition illuminating the corporate-feed network. The main beam can be steered in the H-plane up to $\pm 40^{\circ}$ with scan losses of the order of $2 \mathrm{~dB}$. In a scan range of $\pm 27^{\circ}$, the measured SLLs are lower than $-18.6 \mathrm{~dB}$ at the design frequency. The SLL level is about $-13.26 \mathrm{~dB}$ in the E-plane due to the uniform illumination imposed by the corporate-feed network. The measured antenna gain is about $29 \mathrm{dBi}$ at broadside with an efficiency of $93 \%$ at $29.25 \mathrm{GHz}$. The gain and efficiency are larger than $27.6 \mathrm{dBi}$ and $80 \%$ in the band $27.5-31 \mathrm{GHz}$, respectively. Besides, measurement results in the entire Ka-band demonstrate the wideband operation of the antenna even beyond the targeted Tx Satcom band. The final antenna is made in aluminum in several parts assembled together with screws without using any electromagnetic choke for the metallic junctions. The total thickness of the antenna is $45 \mathrm{~mm}$. The attractive radiation performance, multibeam capability, and small thickness of the structure make such antenna a suitable candidate for next generation Ka-band Satcom terminals for moving platforms or for mobile ground stations.

\section{ACKNOWLEDGMENT}

The authors would like to thank all the staff of the mechanical workshop at IETR and in particular X. Morvan for his contribution during the fabrication of the prototype.

\section{REFERENCES}

[1] R. Pearson, "Next generation mobile SATCOM terminal antennas for a transformed world," in Proc. Eur. Conf. Antennas Propag., Rome, Italy, Apr. 11-15, 2011, pp. 2341-2345. 
[2] L. Diamond, "Ka-band user terminal antennas," presented at 34th ESA Antenna Workshop; 2nd Evol. Satell. Telecommun. Ground Segments Workshop Satcom User Terminal Antennas, Noordwijk, The Netherlands, Oct. 3-5, 2012.

[3] B. Tripodi, F. Di Marca, T. Cadili, C. Mollura, F. Di Maggio, and M. Russo "Ka-band active phased array antenna system for satellite communications on the move terminal," in Proc. Eur. Conf. Antennas Propag., Rome, Italy, Apr. 11-15, 2011, pp. 2628-2630.

[4] M. Shelley, J. Vazquez, and D. Moore, "X- and Ka-band low profile antennas for aeronautical and land mobile Satcom," in Proc. Eur. Conf. Antennas Propag., The Hague, The Netherlands, Apr. 6-11, 2014, pp. 2619-2622.

[5] L. I. Boccia et al., "SATCOM Rx/Tx user terminal antennas with intelligent pixels," in Proc. 34th ESA Antenna Workshop; 2nd Evol. Satell. Telecommun. Ground Segments Workshop Satcom User Terminal Antennas, Noordwijk, The Netherlands, Oct. 3-5, 2012 [Online]. Available: http://telecom.esa.int/telecom/media/document/01_1145_ Boccia.pdf

[6] Kymeta Website. (2014, Sep.) "mTenna ${ }^{\text {TM }}$ and Metamaterials“ [Online]. Available: http://www.kymetacorp.com

[7] W. W. Milroy, "The continuous transverse stub (CTS) array: basic theory, experiment, and application," in Proc. Antenna Appl. Symp., Sep. 25-27, 1991, vol. 2, pp. 253-283.

[8] N. E. Lindenblad and P. Jefferson, "Multiple slot antenna," U.S. Patent 2 628311, Feb. 10, 1953.

[9] W. W. Milroy, "Continuous transverse stub element devices and methods of making same," U.S. Patent 5266 961, Nov. 30, 1993.

[10] W. W. Milroy, "Antenna array configurations employing continuous transverse stub elements," U.S. Patent 5412 394, May 2, 1995.

[11] W. W. Milroy, "Compact, ultra-wideband, antenna feed architecture comprising a multistage, multilevel network of constant reflection-coefficient components," U.S. Patent 6075 494, Jun. 13, 2000.

[12] Y. Xu, H. Dong, Y. Liu, and P. Zhang, "Continuous transverse stub (CTS) array antenna," in Proc. Int. Symp. Antennas Propag., Nagoys, Japan, Mar. 2012, pp. 1083-1086.

[13] A. Neto and J. J. Lee, "Ultrawide-band properties of long slot arrays," IEEE Trans. Antennas Propag., vol. 54, no. 2, pp. 534-543, Feb. 2006.

[14] A. Neto, D. Cavallo, G. Gerini, and G. Toso, "Scanning performances of wideband connected arrays in the presence of a backing reflector," IEEE Trans. Antennas Propag., vol. 57, no. 10, pp. 3092-3102, Oct. 2009.

[15] F. F. Manzillo, M. Ettorre, M. Casaletti, N. Capet, and R. Sauleau, "Active impedance of infinite parallel-fed continuous transverse stub arrays," IEEE Trans. Antennas Propag., vol. 63, no. 7, pp. 3291-3297, Jul. 2015.

[16] M. Ettorre, R. Sauleau, and L. Le Coq, "Multi-beam multi-layer leakywave SIW pillbox antenna for millimeter-wave applications," IEEE Trans. Antennas Propag., vol. 59, no. 4, pp. 1093-1100, Apr. 2011.

[17] W. W. Milroy, S. B. Coppedge, A. Ekmekji, S. Hashemi-Yeganeh, and S. G. Buczek, "True-time-delay feed network for CTS array," U.S. Patent 7432871 , Oct. 7, 2008.

[18] R.I. Wolfson, W. M. Milroy, and S.B. Coppedge, "Methods of fabricating true-time delay continuous transverse stub array," U.S. Patent 5995055 , Aug. 13, 2002.

[19] E. L. Holzman, "Pillbox antenna design for millimeter-wave base-station applications," IEEE Antennas Propag. Mag., vol. 45, no. 1, pp. 27-37, Feb. 2003.

[20] R. S. Robertson, R. T. Kihm, E. L. Holzman, J. Poelker, and R. L. Bowen, "A noncoherent W-band transceiver," in Proc. IEEE MTT-S Int. Microw. Symp., May 25-27, 1988, vol. 2, pp. 1027-1030.

[21] D. M. Pozar, Microwave Engineering, 4nd ed. Hoboken, NJ, USA: Wiley, 2012. ch. 3.

[22] N. Marcuvitz, Waveguide Handbook, 3rd ed. New York, NY, USA: McGraw Hill, 1951.

[23] G. L. Matthaei, E. M. T. Jones, and L. Young, Microwave Filters, Impedance-Matching Networks, and Coupling Structures. New York, NY, USA: McGraw-Hill, 1964.

[24] W. Rotman, "Wide-angle scanning with microwave double-layer pillboxes," IRE Trans. Antennas Propag., vol. 6, no. 1, pp. 96-105, Jan. 1958. 